\title{
SHAPE TRIVIALITY AND METRIC CONTRACTIONS
}

\author{
W. HOLSZTYŃSKI
}

\begin{abstract}
Let $(X, d)$ be a nonempty compact metric space such that for every $\varepsilon>0$ there exists a map $f: X \rightarrow X$ satisfying

(i) $d(x, f(x))<\varepsilon$ for every $x \in X$, and

(ii) $d(f(x), f(y))<d(x, y)$ for every $x, y \in X$.

Then, as proved in this paper, the shape of $X$ is trivial. This improves an earlier result of $K$. Borsuk [1], who proved that, under the same assumptions, $X$ is acyclic.
\end{abstract}

A function $f: X \rightarrow X$ of a metric space $(X, d)$ is called a metric contraction if

$$
d(f(x), f(y))<d(x, y) \quad \text { whenever } x, y \in X, x \neq y .
$$

Answering a question posed by Nadler, Jr. [2], Borsuk proved the following:

THEOREM (BORSUK [1]). Let $(X, d)$ be a compact metric space satisfying the following condition:

(*) for every $\varepsilon>0$ there exists a metric contraction $f: X \rightarrow X$ such that $d(f(x), x)<\varepsilon$ for every $x \in X$.

Then $X$ is acyclic.

In this paper we shall prove a stronger result:

THEOREM 1. Let $(X, d)$ be a compact metric nonempty space satisfying condition (*). Then the shape of $X$ is trivial.

First let us observe that if $f: X \rightarrow X$ is a metric contraction of a compact metric space $X$, then $f(A)=A$ can hold for a closed subset $A$ of $X$ only if $A$ has at most one point. Thus the following theorem is more general than Theorem 1 .

THEOREM 2. Let $X$ be a compact Hausdorff nonempty space satisfying the following condition:

(**) for every neighbourhood $U$ of $\Delta_{X}=\{(x, x): x \in X\}$ in $X \times X$ there exists a $U$-shift $f: X \rightarrow X$ such that if $f(A)=A$ for a closed subset $A$ of $X$ then $A$ has at most one point.

Then $X$ has trivial shape. $(f: X \rightarrow X$ is called a $U$-shift if $f$ is continuous and $(x, f(x)) \in U$ for every $x \in X$.

Received by the editors March 28, 1977.

AMS (MOS) subject classifications (1970). Primary 55D99, 54E40; Secondary 54H25.

Key words and phrases. Shape theory, trivial shape, metric contractions approximating the identity map, acyclicity, fixed point.

(1) American Mathematical Society 1978 
Proof. We have to show that arbitrary continuous map $g: X \rightarrow P$ of $X$ into an arbitrary finite polyhedron is homotopic to a constant map.

Let $V$ be a neighbourhood of $\Delta_{P}$ in $P \times P$ such that continuous maps $g_{1}$, $g_{2}: X \rightarrow P$ are homotopic whenever $\left(g_{1}(x), g_{2}(x)\right) \in V$ for all $x \in X$. Since finite polyhedron $P$ is an ANR, such $V$ exists. Next, let $U=(g \times g)^{-1}(V)$, where $g: X \rightarrow P$ is a continuous map. Consider a map $f: X \rightarrow X$ given by (**), and the family $F$ of all closed nonempty subsets $A$ of $X$ such that:

(i) $f(A) \subseteq A$,

(ii) for every neighbourhood $W$ of $A$ in $X$ there exists a map $j: X \rightarrow X$ such that $j(X) \subseteq W$ and $g \circ j$ is homotopic to $g: X \rightarrow P$.

Obviously $X \in F$. It is also easy to see that the intersection of a chain of $F$ belongs to $F$. Thus, by the Kuratowski-Zorn theorem, $F$ has a minimal member $A_{0}$.

Let $W$ be an arbitrary neighbourhood of $f\left(A_{0}\right)$ in $X$. Let $W_{0}=f^{-1}(W)$. Then there exists $j: X \rightarrow X$ such that $j(X) \subseteq W_{0}$ and $g \circ j$ is homotopic to $g$. Since

$$
(j(x), f \circ j(x)) \in U \text { for every } x \in X,
$$

hence $g \circ f \circ j$ is homotopic to $g \circ j$, i.e. $f \circ j$ is a map such that $(f \circ j)(X) \subseteq$ $W$ and $g \circ(f \circ j)$ is homotopic to $g$. This means that (ii) holds for $A=f\left(A_{0}\right)$. Also

$$
f(A)=f\left(f\left(A_{0}\right)\right) \subseteq f\left(A_{0}\right)=A .
$$

Thus $f\left(A_{0}\right) \in F$. Since $f\left(A_{0}\right) \subseteq A_{0}$ and $A_{0}$ is a minimal member of $F$, hence $f\left(A_{0}\right)=A_{0}$. By (**), $A_{0}=\{a\}$ is a one-point set (i.e. $a$ is the unique fixed point of $f)$. Let $W_{1}=\{x \in X:(a, x) \in U\}$ and $j: X \rightarrow X$ be such that $j(X) \subseteq W_{1}$ and $g \circ j$ is homotopic to $g$. But $g \circ j: X \rightarrow P$ is homotopic also to the constant map $x \mapsto g(a)$. The theorem is proved.

REMARK. Our considerations above may be easily applied to obtain the following simple facts:

1. If $X$ is a nonempty compact Hausdorff space and $f: X \rightarrow X$ is a continuous map, then there exists a nonempty closed subset $A$ of $X$ such that $f(A)=A$ and $f(B)$ is not contained in $B$ for any proper closed subset $B$ of $A$.

2. If $f: X \rightarrow X$ is a metric contraction of a compact metric space $X$ then there exists a unique $a \in X$ such that $f(a)=a$. Furthermore, the sequence $y$, $f(y), f^{2}(y), \cdots$ is convergent to this unique fixed point $a$ for every $y \in X$.

Indeed, let $Y$ be the set of all limit points of the sequence $y, f(y)$, $f^{2}(y), \ldots$ Then $f(Y)=Y$. Thus $Y=\{a\}$ and $a=\lim _{n} f^{n}(y)$.

\section{REFERENCES}

1. K. Borsuk, Concerning a problem due to Sam B. Nadler, Jr., Colloq. Math. 35 (1976), 63-65.

2. S. B. Nadler, Jr., Some problems concerning stability of fixed points, Colloq. Math. 27 (1973), 263-268.

Department of Mathematics, Untversity of Western Ontario, London, Ontario, Canada

Current address: 2927 Burlington Court, Ann Arbor, Michigan 48105 